Artículo científico

Volumen 32(2):479-486. Mayo-agosto, 2021

e-ISSN 2215-3608, doi:10.15517/am.v32i2.44057

https://revistas.ucr.ac.cr/index.php/agromeso/index

\title{
Relación entre enfermedades y rendimiento de granos de soja ${ }^{1}$
}

\section{Relationship among diseases and grain yield in soybean}

\author{
M. Lavilla ${ }^{2}$ A. Ivancovich ${ }^{2}$
}

1 Recepción: 6 de octubre, 2020. Aceptación: 4 de febrero, 2021. Este trabajo formó parte de un proyecto de extensión N Exp. 2563/17 financiado por la Universidad Nacional del Noroeste de la provincia de Buenos Aires (UNNOBA).

2 Universidad Nacional del Noroeste de la provincia de Buenos Aires, Monteagudo 2772 (2700), Pergamino, Buenos Aires, Argentina. miguellavillapergamino@gmail.com (autor para la correspondencia https://orcid.org/0000-0002-7282-4696), ajivancovich@comunidad. unnoba.edu.ar (https://orcid.org/0000-0002-4195-2986).

\section{Resumen}

Introducción. Las enfermedades vasculares y del follaje se encuentran entre los factores más relevantes que afectan la producción de soja (Glycine max). Objetivo. Cuantificar los daños de las enfermedades vasculares y del follaje sobre el rendimiento en el cultivo de soja. Materiales y métodos. Este estudio se llevó a cabo durante dos años consecutivos (2018 y 2019) en la localidad de Pergamino, Buenos Aires, Argentina. Para el caso de las enfermedades vasculares el muestreo se realizó sobre cuarenta lotes de la localidad de Pergamino. En cada lote se recolectaron treinta pares de plantas con base en la enfermedad presente. Cada par de planta se cosechó a mano y se cuantificó el número de granos por planta (NGP) y el rendimiento por planta (RP). Para la cuantificación de las pérdidas de rendimiento por las enfermedades del follaje se realizaron ensayos con y sin fungicida foliar. En cada tratamiento se determinó el rendimiento $\left(\mathrm{kg} \mathrm{ha}^{-1}\right)$ y sus principales componentes. Resultados. La podredumbre húmeda del tallo causada por Sclerotinia sclerotiorum fue la enfermedad que generó los mayores daños en el NGP y el RP. Las enfermedades vasculares provocaron daños en el cultivo mayores al $50 \%$. Las enfermedades del follaje en soja redujeron significativamente el rendimiento entre un $11 \%$ a un $20 \%$, cuando no fueron controladas con fungicidas foliares. Conclusión. Las enfermedades vasculares y del follaje diagnosticadas en este estudio redujeron significativamente el rendimiento y sus componentes en el cultivo de soja.

Palabras claves: fungicidas foliares, enfermedades vasculares, enfermedades del follaje

\begin{abstract}
Introduction. Vascular and foliage diseases are among the most relevant factors affecting soybean production. Objective. To quantify the damage of vascular and foliage diseases on the yield of soybean (Glycine max) crops. Materials and methods. This study was carried out for two consecutive years (2018 and 2019) in the town of Pergamino Buenos Aires, Argentina. In the case of vascular diseases, the sampling was carried out on 40 lots from the town of Pergamino. In each batch, 30 pairs of plants were collected by relieved disease. Each pair of plants was harvested by hand quantifying the number of grains per plant (NGP) and the yield per plant (RP). For the
\end{abstract}


quantification of yield losses due to foliage diseases, tests were carried out with and without foliar fungicide. In each treatment the yield $\left(\mathrm{kg} \mathrm{ha}^{-1}\right)$ and its main components were determined. Results. The wet rot of the stem caused by Sclerotinia sclerotiorum was the disease that generated the greatest damage losses in the NGP and RP. Vascular diseases can cause damage losses in the crop yield per plant, greater than $50 \%$ and this must be associated with the percentage of incidence of the disease or diseases present in the soybean lot to determine the total damage losses. Foliage diseases in soybean can significantly reduce the yield between $11 \%$ to $20 \%$, if they are not controlled in time with foliar fungicides. Conclusion. The vascular and foliage diseases diagnosed in this study significantly reduced the yield and its components in the soybean crop.

Keywords: foliar fungicides, vascular diseases, foliage diseases.

\section{Introducción}

La soja [Glycine $\max ($ L.) Merrill] es la principal oleaginosa en la República de Argentina, con una superficie sembrada de diecisiete millones de hectáreas, una producción de casi cuarenta millones de toneladas y un rendimiento promedio de $2233 \mathrm{~kg} \mathrm{ha}^{-1}$ (Estimaciones Agrícolas, 2016). Las provincias con mayor producción de soja son Buenos Aires, Córdoba, Entre Ríos y Santa Fe (Estimaciones Agrícolas, 2016).

Las enfermedades se encuentran entre los factores más relevantes que afectan la producción de soja. El cultivo de esta leguminosa es afectado por las enfermedades desde la siembra hasta la cosecha, incluido el período de almacenamiento. Los daños en rendimiento de grano provocados por las enfermedades a nivel mundial varían entre 10 y $15 \%$ (Bandara et al., 2020; Wrather et al., 2001; Wrather \& Koenning, 2009).

En Argentina, los daños causados por las enfermedades en el cultivo de soja varían entre un 10 y un $30 \%$ (Vallone, 2003), lo que equivale a una pérdida de más de US\$ 1000 millones por año.

Durante las últimas décadas diferentes enfermedades han causado problemas sanitarios en el cultivo de soja en la Argentina. En la década de los ochenta fue la podredumbre húmeda causada por Sclerotinia sclerotiorum (Ivancovich et al., 1981; Sillón, 2006), la muerte de plántulas por damping off (causada por Rhizoctonia solani y Fusarium spp.) y los problemas de calidad de semillas causados por Phomopsis sojae, Colletotrichum spp., y Fusarium spp. (Pioli, 2000). Durante la década de los noventa la soja fue afectada por el síndrome de la muerte repentina (Fusarium tucumaniae, F. virguliforme y otras especies de Fusarium) y el cancro del tallo causado por Phomopsis phaseoli var. meridionalis (Ivancovich, 1992; Pioli et al., 1993; 1997).

En la última década las denominadas enfermedades de fin de ciclo (EFC) son las más frecuentes en el cultivo de soja en Argentina, las cuales han causado pérdidas de rendimiento en granos de hasta un $30 \%$ (Ivancovich, 1998; Ploper, 1999; Vallone \& Giorda, 1997). El complejo de las EFC está constituido principalmente por la mancha ojo de rana causada por Cercospora sojina Hara (Ivancovich et al., 1999; Ploper, 1999), la mancha marrón de la hoja causada por Septoria glycines Hemmi y el tizón foliar por Cercospora causado por Cercospora kikuchii T. Matsumoto y Tomoyasu (Ivancovich et. al., 2018; Lavilla et. al., 2018). La mancha ojo de rana causó una epifítia importante en los ciclos agrícolas de soja 2009/10 y 2010/11, las pérdidas de rendimiento superiores a los $2300 \mathrm{~kg}$ ha $^{-1}$ en cultivares susceptibles (Distéfano \& Gadbán, 2007).

El objetivo de este estudio fue cuantificar los daños de las enfermedades vasculares y del follaje sobre el rendimiento en el cultivo de soja.

${ }^{3}$ Complejo de enfermedades que afectan a los cultivos en estados fenológicos iniciales. 


\section{Materiales y métodos}

\section{Ubicación del relevamiento}

Para cuantificar los daños (Zadoks, 1985) sobre el rendimiento en soja (Glycine max) por causa de las principales enfermedades vasculares y del follaje, se llevó a cabo un estudio durante dos años consecutivos (2018 y 2019) en la localidad de Pergamino, Buenos Aires, Argentina. Para las enfermedades vasculares el muestreo se realizó sobre cuarenta lotes comerciales, los cuales presentaban un paquete tecnológico adecuado para el control de artrópodos y malezas. Los ensayos para analizar el efecto de las enfermedades del follaje sobre el rendimiento se realizaron en dos lotes comerciales. Los estudios fitopatológicos se realizaron en el laboratorio de la Universidad Nacional de Noroeste de la provincia de Buenos Aires (UNNOBA), Pergamino, Argentina.

\section{Diagnóstico de los agentes causales de las enfermedades}

El diagnóstico de los agentes causales de las enfermedades se realizó bajo microscopio estereoscópico y óptico. En caso de incertidumbre del agente causal de la enfermedad, a las muestras extraídas del campo se las desinfectó con hipoclorito de sodio al $2 \%$, luego se las enjuagaron con agua estéril y, posteriormente, se las sometió a cámara húmeda durante $48 \mathrm{~h}$.

\section{Cuantificación de los daños sobre el rendimiento en soja por causa de las enfermedades vasculares.}

En cada lote se recolectaron treinta pares de plantas por enfermedad relevada. Se geo posicionaron en el lote con estacas los pares de plantas, que se componían de una planta sana y una planta enferma, las cuales se etiquetaron cuando se encontraban en el estado fenológico de $\mathrm{R}_{5}$ (semillas de $3 \mathrm{~mm}$ de largo en una vaina en alguno de los cuatro nudos superiores con una hoja totalmente desarrollada) (Fehr et al., 1971). La cosecha de los pares de plantas se realizó en estado fenológico $\mathrm{R}_{8}$ (un $95 \%$ de las vainas han alcanzado su color típico de madurez) (Fehr et al., 1971). Finalmente, los pares de plantas previamente identificados, se llevaron al laboratorio de Fitopatología de la UNNOBA, en donde se cosecharon a mano y se determinó el número de granos por planta (NGP) y el rendimiento por planta (RP). El RP se obtuvo con base en el peso de los granos presentes en la planta con una balanza digital de precisión 0,01g. Los datos relevados en los dos años (2018-2019) se promediaron y analizaron a través de una prueba de t pareada $(\mathrm{p} \leq 0,05)$, utilizando el programa estadístico InfoStat (Di Renzo et al., 2011).

\section{Cuantificación de los daños sobre el rendimiento en soja por causa de las enfermedades del follaje.}

Se realizaron dos ensayos en dos lotes diferentes, uno con elevada cantidad de mancha marrón (MM) de la soja (Septoria glycines) y el otro con valores significativos del tizón foliar por Cercospora (TFC) (Cercospora kikuchii).

La MM se cuantificó utilizando la variable patométrica altura de la planta con síntomas (APS) (Larrañaga et al., 2018). En cambio, el TFC se obtuvo a partir de la incidencia (I) y la severidad (S) (Agrios, 2005).

Durante los dos años de evaluación se utilizó un diseño en bloques completos al azar con tres repeticiones y con dos tratamientos, uno con fungicida foliar (fluxapyroxad $5 \%$, pyraclostrobin $8,1 \%$ y epoxiconazole $5 \%$ ) y otro sin fungicida foliar; la unidad experimental fue de $18 \mathrm{~m}^{2}$. Cuando las enfermedades llegaron a su umbral de acción, $25 \%$ de la APS para la MM (Ivancovich \& Couretot, 2009) y $50 \%$ de I y $25 \%$ de S para el TFC (Lavilla et al., 2019); se realizó la aplicación de un fungicida foliar a una dosis de $800 \mathrm{ml} \mathrm{ha}^{-1}$ con una mochila de presión constante, equipada con una barra de cuatro pastillas de cono hueco distanciadas a $35 \mathrm{~cm}$, arrojando un caudal de 
1701 ha $^{-1}$, con una presión de $50 \mathrm{Ib} \mathrm{pulg}^{-2}$. La cosecha los tratamientos y repeticiones se realizó en estado fenológico $\mathrm{R}_{8}$. El rendimiento de grano $(\mathrm{R})\left(\mathrm{kg} \mathrm{ha}^{-1}\right)$ se cuantificó con base en el peso total de los granos cosechados en los $16 \mathrm{~m}^{2}$ (descontado el efecto bordura). El peso de mil granos (PMG) se determinó, primero cuantificando los 1000 granos con un contador electrónico y luego pesando estos granos en una balanza de precisión. El número de granos por $\mathrm{m}^{2}(\mathrm{NG})$ se definió relacionando el peso total de la muestra con el PMG. Para un estudio general de las variables cuantitativas se realizó un análisis de la varianza (ANAVA) y la comparación de medias mediante una prueba de Tukey $(a l f a=5 \%)$. Se corroboraron los supuestos de normalidad de distribución, dos errores mediante la prueba de Shapiro-Wilks modificado y de homogeneidad de varianzas mediante el gráfico de residuos versus predichos, utilizando el programa estadístico InfoStat (Di-Renzo et al., 2011).

\section{Resultados}

\section{Cuantificación de los daños sobre el rendimiento en soja por causa de las enfermedades vasculares}

En el relevamiento se pudieron diagnosticar las siguientes enfermedades vasculares: la podredumbre húmeda del tallo (PHT) causada por Sclerotinia sclerotiorum, el síndrome de la muerte repentina (SMR) causado por Fusarium tucumaniae, la podredumbre carbonosa (PC) causada por Macrophomina phaseolina y al cancro del tallo (CT) causado por Diapothe caulivora. Los daños ocasionados de las enfermedades vasculares sobre el rendimiento de soja detalladas se presentan en el Cuadro 1.

La PHT fue la enfermedad que generó más daños sobre el número de granos por planta (NGP) y el rendimiento promedio por planta (RP) (Cuadro 1). En cambio, el SMR, la PC y el CT provocaron daños similares en las variables cuantitativas analizadas (Cuadro 1 ).

Cuadro 1. Promedio del número de granos por planta (NGP) y el rendimiento por planta (RP) y su relación con algunas de las enfermedades vasculares que afectan al cultivo de soja (Glycine max). Pergamino, Argentina (2018-2019).

Table 1. Average seed number per plant (NGP) and yield per plant (PR) and its relationship with some of the vascular diseases that affect soybean (Glycine max) crops. Pergamino, Argentina (2018-2019).

\begin{tabular}{cccc}
\hline Enfermedad* & Planta & NGP & RP \\
\hline PHT & Sana & $90,54 \mathrm{~A}$ & $16,07 \mathrm{~A}$ \\
PHT & Enferma & $39,95 \mathrm{~B}$ & $6,44 \mathrm{~B}$ \\
SMR & Sana & $104,23 \mathrm{~A}$ & $18,23 \mathrm{~A}$ \\
SMR & Enferma & $57,38 \mathrm{~B}$ & $10,94 \mathrm{~B}$ \\
PC & Sana & $89,11 \mathrm{~A}$ & $14,01 \mathrm{~A}$ \\
PC & Enferma & $47,03 \mathrm{~B}$ & $7,72 \mathrm{~B}$ \\
CT & Sana & $91,55 \mathrm{~A}$ & $15,23 \mathrm{~A}$ \\
CT & Enferma & $44,23 \mathrm{~B}$ & $8,44 \mathrm{~B}$ \\
\hline
\end{tabular}

* Podredumbre húmeda del tallo (PHT) causada por Sclerotinia sclerotiorum, síndrome de la muerte repentina (SMR) causada por Fusarium tucumaniae. Podredumbre carbonosa (PC) causada por Macrophomina phaseolina y cancro del tallo (CT) causado por Diapothe caulivora /* Sclerotinia stem rot caused by Sclerotinia sclerotiorum (PHT), Sudden death syndrome (SMR) caused by Fusarium tucumaniae, Charcoal rot (PC) caused by Macrophomina phaseolina, and Stem canker (CT) caused by Diapothe caulivora. 


\section{Cuantificación de los daños sobre el rendimiento en soja por causa de las enfermedades del follaje}

La cantidad de mancha marrón (MM) y el tizón foliar por Cercospora (TFC) redujeron significativamente el rendimiento y sus componentes en el cultivo de soja, cuando ambas enfermedades no se controlaron con fungicida foliar (Cuadro 2). No controlar la mancha marrón al $25 \%$ de altura de la planta con síntomas de la enfermedad, redujo el rendimiento en hasta un $15 \%$ (Cuadro 2). Los daños causados por el TFC sobre el cultivo de soja redujeron el rendimiento en un $11 \%$ (Cuadro 2) en aquellos tratamientos que superaron el $50 \%$ de I y $25 \%$ de S para el TFC y no se les aplicó el fungicida foliar.

Cuadro 2. Promedio del número de granos por $\mathrm{m}^{2}(\mathrm{NG})$, el peso de mil granos (PMG) en gramos y rendimiento $(\mathrm{R})$ en $\mathrm{kg}$ ha ${ }^{-1}$ y su relación con algunas de las enfermedades del follaje que afectan al cultivo de soja (Glycine max). Pergamino, Argentina (2018-2019). Table 2. Average seed number per $\mathrm{m}^{2}$ (NG), thousand seed weight (PMG) in grams and yield (R) in $\mathrm{kg} \mathrm{ha}^{-1}$ and its relationship with some of the foliage diseases that affect soybean crops. Pergamino, Argentina (2018-2019).

\begin{tabular}{ccccc}
\hline Enfermedad* & Fungicida foliar $(\mathbf{F F})$ & NG** & PMG*** & R**** \\
\hline MM & Sin FF & $2521,43 \mathrm{~A}$ & $140,01 \mathrm{~A}$ & $3530,20 \mathrm{~A}$ \\
MM & Con FF & $2606,25 \mathrm{~B}$ & $160,08 \mathrm{~B}$ & $4168,21 \mathrm{~B}$ \\
TFC & Sin FF & $2407,19 \mathrm{~A}$ & $167,03 \mathrm{~A}$ & $4020,54 \mathrm{~A}$ \\
TFC & Con FF & $2475,40 \mathrm{~B}$ & $183,44 \mathrm{~B}$ & $4530,37 \mathrm{~B}$ \\
\hline
\end{tabular}

* mancha marró (MM) de la soja causada porSeptoria glycines. Tizón foliar por Cercospora (TFC) causado por Cercospora kikuchii. **número de granos por $\mathrm{m}^{2}(\mathrm{NG})$, ***el peso de mil granos (PMG) en gramos y ****el rendimiento $(\mathrm{R})$ en $\mathrm{kg}^{-1} / * \mathrm{Br}^{-1}$ Brown spot (MM) caused by Septoria glycines, Cercospora leaf blight (TFC) caused by Cercospora kikuchii, **number of grains per $\mathrm{m}^{-2}$ (NG), $* * *$ thousand seed weight $(\mathrm{PMG})$ in $* * * *$ grams and yield $(\mathrm{R})$ in $\mathrm{kg} \mathrm{ha}^{-1}$.

\section{Discusión}

Las enfermedades vasculares y las enfermedades del follaje diagnosticadas redujeron significativamente el rendimiento en granos en el cultivo de soja. Según un estudio, la podredumbre húmeda del tallo (PHT) y el cancro del tallo (CT) disminuyeron el rendimiento por planta (RP) en un 65,5\% y en un $50 \%$, respectivamente (Montoya et al., 2017). Estos resultados fueron similares a los relevados en la presente investigación; en ese sentido, Lenzi et al. (2011) observaron daños sobre el rendimiento en el cultivo de soja por causa del síndrome de la muerte repentina (SMR) de hasta un 58,5\% (Lenzi et al., 2011). En esta experiencia, llevada a cabo durante dos años consecutivos (2018 y 2019), se observaron daños sobre el RP promedio de casi un $40 \%$ por causa del SMR. Daños que se calcularon asociando el RP con el porcentaje de incidencia (número de plantas enfermas entre número total de plantas analizadas por cien) de la o las enfermedades en el lote en estudio.

El número de granos por planta (NGP) también se redujo significativamente en las plantas de soja afectadas por las enfermedades vasculares. En promedio se observó que las enfermedades vasculares provocaron daños de casi un $50 \%$ sobre el NGPen el cultivo de soja, estos resultados coinciden con los observados por Lenzi et al. (2011) y Montoya et al. (2017) en el mismo cultivo.

Las enfermedades de fin de ciclo (EFC), causan una defoliación prematura en el cultivo de soja que reduce la eficiencia de intercepción de la radiación, la tasa de crecimiento en el período crítico y, por ende, el rendimiento en granos en el cultivo de soja. La aplicación de diferentes fungicidas foliares redujo de manera significativa la severidad de la MM y aumentó el rendimiento de grano (R) en casi un $20 \%$ en el cultivo de soja (Zárate, 2017). Además, si no se controla a tiempo el TFC con fungicidas foliares las pérdidas de $\mathrm{R}$ en el cultivo de soja pueden 
llegar a ser del $11 \%$ (Lavilla et al., 2019). Los resultados analizados en esta investigación demostraron que el TFC redujo en un $11 \%$ el $\mathrm{R}$ en el cultivo de soja, al no aplicar un fungicida foliar.

Tanto el NG como el PMG fueron afectados por las EFC observadas en esta experiencia, lo cual explica las pérdidas de $\mathrm{R}$ causadas por las enfermedades del follaje en el cultivo de soja.

Este es el primer trabajo donde se documentan las pérdidas de rendimiento causadas por enfermedades vasculares y del follaje en el cultivo de soja en el norte de la provincia de Buenos Aires (Argentina) durante dos años consecutivos, 2018 y 2019.

\section{Conclusiones}

Las enfermedades vasculares y del follaje diagnosticadas en este estudio redujeron significativamente el rendimiento y sus componentes en el cultivo de soja. Si se considera a las enfermedades vasculares como único factor biótico que afectó al cultivo, estas causaron pérdidas de rendimiento por planta mayores al $50 \%$. Las enfermedades del follaje en soja disminuyeron significativamente el rendimiento entre un $11 \%$ a un $20 \%$, cuando las mismas no se controlaron con fungicidas foliares.

\section{Referencias}

Agrios, G. N. (2005). Plant Pathology (5 ${ }^{\text {th }}$ Ed.). Academic Press.

Bandara, A. Y., Weerasooriya, D. K., Bradley, C. A., Allen, T. W., \& Esker, P. D. (2020). Dissecting the economic impact of soybean diseases in the United States over two decades. PLOS ONE, 15(4), Article e0231141. https://doi.org/10.1371/ journal.pone.0231141

Di-Rienzo J. A., Casanoves F., Balzarini M. G., González L., Tablada M., \& Robledo C. W. (2011). InfoStat. Grupo InfoStat. http://www.infostat.com.ar

Distéfano, S., \& Gadbán, L. (2007). Panorama fitopatológico del cultivo de soja en la campaña 2006/2007. Instituto Nacional de Tecnología Agropecuaria. https://docplayer.es/31146768-Panorama-fitopatologico-del-cultivo-de-soja-en-lacampana.html

Estimaciones Agrícolas. (2016, 29 abril). Estimaciones Agrícolas. https://datosestimaciones.magyp.gob.ar/reportes. php?reporte=Estimaciones

Fehr, W. R., Caviness, C. E., Burmood, D. T., \& Pennington, J. S. (1971). Stage of development descriptions for soybeans, Glycine max (L.) Merrill 1. Crop Science, 11(6), 929-931. https://doi.org/10.2135/cropsci1971.0011183x001100060 $051 \mathrm{x}$

Ivancovich, A. (1992). Aparición del cancro del tallo en cultivos de soja en el área de la EEA Pergamino (No 10). Instituto Nacional de Tecnología Agropecuaria.

Ivancovich, A. (1998, 15 abril). Diagnóstico y manejo de enfermedades en soja [Presentación en conferencia]. IV Curso de diagnóstico y manejo de enfermedades de soja, Pergamino, Argentina.

Ivancovich, A., Botta, G., \& Annone, J. (Eds.). (1999). Enfermedades fúngicas de soja en madurez en el partido de Pergamino (Bs. As-Argentina). Congreso Mundial de Soja. 
Ivancovich, A., \& Couretot, L. (Eds.). (2009, august 25-28). Diagrammatic scale for evaluation of brown spot on soybean [Conference presentation abstract]. World Soybean Conference Research: Developing a Global Soy Blueprint for a Safe Secure and Sustainable Supply, Beijing, China.

Ivancovich, A., Lavilla, M., Martínez, M., Moschini, R., Díaz Paleo, A., Sosa, C., Izaguirre, S., Delorenzi, V., Simón, J., \& Cauda, N. (2018). Prevalencia y severidad de la mancha marrón de la soja causada por Septoria glycines en el norte de la provincia de Buenos Aires. Universidad Nacional de Tucumán.

Ivancovich, A., Martinez, C., \& Sarlo, O. (1981). Pérdidas ocasionadas por Sclerotinia slcerotiorum en el partido de Pergamino en el ciclo 1980/81. Instituto Nacional de Tecnología Agropecuaria.

Larrañaga, J., Ivancovich, A., \& Lavilla, M. (Eds.). (2018). Evaluación del momento de aplicación de fungicidas foliares sobre el desarrollo de la "mancha marrón” de la hoja, causada por Septoria glycines Hemmi, en soja. Universidad Nacional del Noroeste de la provincia de Buenos Aires.

Lavilla, M., Ivancovich, A., \& Díaz Paleo, A. (2019). Umbral de daño económico para el tizón foliar por Cercospora causado por Cercospora kikuchii en soja. MERCOSOJA.

Lavilla, M., Ivancovich, A., Díaz Paleo, A., Sosa, C., Cauda, N., Izaguirre, S, Delorensi, V., \& Simón J. (Ed.). (2018). Estudios patométricos del tizón foliar por Cercospora causado por Cercospora kikuchii en soja en el norte de la provincia de Buenos Aires. Universidad Nacional de Tucumán.

Lenzi, L. G., Scandiani, M., Distéfano, S., \& Salinas, L. (2011). Efecto del síndrome de la muerte súbita sobre el rendimiento de plantas de soja. Instituto Nacional de Tecnología Agropecuaria. https://inta.gob.ar/sites/default/files/script-tmp-intaefecto_del_sndrome_de_la_muerte_sbita_sobre_soja.pdf

Montoya, M., Schlie, G., \& Ridao, A. C. (Eds.). (2017). Cuantificación de pérdidas de rendimiento por Sclerotinia sclerotiorum y Diaporthe caulivora en soja. Asociación Argentina de Fitopatología.

Pioli, R. (Ed.). (2000). Estudio epidemiológico de patógenos fúngicos asociados a semillas y frutos de soja. Asociación Latinoamericana de Fitopatología.

Pioli, R., Gattuso, S., Prado, D., \& Borghi, A. (1997). Recent outbreak of stem canker (Diaporthe phaseolorum var. meridionalis) of soybean in Santa Fe, Argentina. Plant Disease, 81(10), Article 1215. https://doi.org/10.1094/pdis.1997.81.10.1215a

Pioli, R., Porfiri, A., Díaz, M., Incremona, M., \& Morata, M. (Eds.). (1993). Determinación de enfermedades fúngicas en soja (Glycine max). Estudio previo a la evaluación de prácticas de manejo. Sociedad de Biología de Rosario.

Ploper, L. D. (Ed.). (1999). Management of economically important diseases of soybean in Argentina. World Soybean Research Conference.

Sillón, M. (2006). Progreso temporal y espacial de las principales enfermedades de fin de ciclo de la soja y su control con fungicidas en el ciclo 2005/2006 (N.o 106). Instituto Nacional de Tecnología Agropecuaria. http://rafaela.inta.gov.ar/ info/miscelaneas/106/misc106_147.pdf

Vallone, S. (2003). Enfermedades de la soja. En E. Satorre (Ed.), El libro de la soja (1a ed., Vol. 1, pp. 123-132). Servicios y marketing agropecuario.

Vallone, S., \& Giorda, L. M. (1997). Enfermedades de la soja en la Argentina. Instituto Nacional de Tecnología Agropecuaria.

Wrather, J. A., Anderson, T. R., Arsyad, D. M., Tan, Y., Ploper, L. D., Porta-Puglia, A., Ram, H. H., \& Yorinori, J. T. (2001). Soybean disease loss estimates for the top ten soybean-producing counries in 1998. Canadian Journal of Plant Pathology, 23(2), 115-121. https://doi.org/10.1080/07060660109506918 
Wrather, A., \& Koenning, S. (2009). Effects of diseases on soybean yields in the United States 1996 to 2007. Plant Health Progress, 10(1), Article 401. https://doi.org/10.1094/php-2009-0401-01-rs

Zadoks, J. C. (1985). On the conceptual basis of crop loss assessment: The threshold theory. Annual Review of Phytopathology, 23(1), 455-473. https://doi.org/10.1146/annurev.py.23.090185.002323

Zárate, J. F. (2017). Evaluación de la aplicación de fungicidas en la severidad de las enfermedades de fin de ciclo en soja y su impacto en los rendimientos [Tesis de grado, Universidad Nacional de La Plata]. Repositorio de la Universidad Nacional de La Plata. http://sedici.unlp.edu.ar/bitstream/handle/10915/61016/Documento_completo__. pdf?sequence $=1 \&$ isAllowed $=\mathrm{y}$ 